\title{
PERCEPCIONES Y EFECTOS DE CAMBIO CLIMÁTICO EN GRUPOS INDÍGENAS DE LA AMAZONÍA COLOM BIANA
}

\author{
Juan Alvaro ECHEVERRI ${ }^{1}$
}

1 Profesor Asociado, Instituto Amazónico de Investigaciones IMANI, Universidad Nacional de Colombia, Sede Amazonía. Kilómetro 2 Vía Tarapacá, Leticia, Amazonas, Colombia. Correo electrónico: jaecheverrir@ unal.edu.co

\section{RESUMEN}

Este artículo presenta una descripción de los principales cambios recientes en el clima como son percibidos por pueblos indígenas del suroriente de la Amazonía colombiana y su correlación con datos climáticos de 8 estaciones meteorológicas en las cuencas de los ríos Caquetá, Putumayo y Amazonas. Se presentan y discuten percepciones y datos sobre incremento de temperatura, cambios en los niveles de precipitación, alteraciones de la estacionalidad en los niveles del río, y efectos de estos cambios en la reproducción de la fauna silvestre, el sistema hortícola y la salud humana, teniendo en cuenta criterios de género, edad y situación territorial de los grupos indígenas.

PALABRAS CLAVES: cambio climático, Amazonía colombiana, pueblos indígenas

\section{PERCEPTIONS AND EFFECTS OF CLIMATE CHANGE ON INDIGENOUS GROUPS OF THE COLOM BIAN AM AZON}

\begin{abstract}
This article presents a description of the main recent effects of changes in climate, as they are perceived by indigenous groups of south-eastern Colombian Amazon, and its correlation with climate data from 8 meteorological stations on the Caqueta, Putumayo and Amazonas River basins. Local perceptions and climate data are presented and discussed on increase in temperatures, changes in the regimes of precipitation, alterations in river level seasonality, and the effects of these changes on the reproduction of wildlife, the horticultural systems and human health, taking into account criteria of gender, age and territorial situation of the indigenous groups.
\end{abstract}

KEYW ORDS: climate change, Colombian Amazon, indigenous peoples. 


\section{INTRODUCCIÓN}

Los indígenas amazónicos son constantes observadores de los ritmos naturales. Sus sistemas de vida -horticultura de tumba y quema, pesca, cacería y recolección de frutos y materiales silvestres- están muy ligados a la sucesión de estaciones bien establecidas. Los indígenas conocen índices ecológicos complejos que ordenan la interrelación de los ciclos del agua, los vientos y la temperatura, y de sus efectos en los ciclos reproductivos de los peces, la fauna terrestre y los frutales silvestres, y en la regulación de las actividades rituales y productivas de la gente. Aunque es normal que la sucesión estacional presente variaciones interanuales, según varios indígenas entrevistados en diferentes áreas de la Amazonía colombiana, los signos e índices naturales que en los últimos años (primera década del siglo XXI) han percibido son "alarmantes": las estaciones están ocurriendo fuera del tiempo, los pulsos de inundación y descenso de los ríos están des-sincronizados con la maduración de los frutos silvestres y el calor está aumentando, entre otros eventos. Más que el impacto del incremento de la temperatura ambiente, son los cambios en la precipitación y la estacionalidad los que tienen mayor impacto en las actividades de subsistencia, principalmente en la horticultura, la reproducción de los peces y en la salud humana, como lo muestro adelante.

El régimen de precipitación de la cuenca amazónica depende de la temperatura superficial del mar (Salick \& Byg, 2007) y de la interacción mar-bosque (Bunyard, 2008). Los bosques tropicales ya han sido profundamente afectados por cambios climáticos causados por la oscilación meridional de El Niño y La Niña. Estos efectos sobre la cuenca amazónica son además exacerbados por la tala de árboles y la fragmentación del bosque (Bunyard, 2008).

En este artículo documento la percepción y los efectos de cambios climáticos recientes en algunos grupos indígenas de la Amazonía noroccidental. Las informaciones y observaciones aquí consignadas hacen referencia principalmente a la percepción de eventos de cambio climático por grupos indígenas del Trapecio amazónico colombiano (en la frontera Brasil-Perú-Colombia) y del interfluvio CaquetáPutumayo (en la frontera amazónica Colombia-Perú). Una percepción generalizada entre estos grupos es que desde la década de los 1990s se han hecho evidentes alteraciones en el clima, particularmente en la estacionalidad, y estos cambios se han vuelto particularmente ostensibles desde el año 2005.

\section{ATERIALES Y MÉTODOS}

Utilizamos métodos cualitativos y cuantitativos para un acercamiento preliminar de la percepción y los efectos de cambios climáticos recientes. Por una parte, en el año 2008, se hizo un conjunto de entrevistas semi-estructuradas a 18 personas, hombres y mujeres, de 10 comunidades en tres regiones de la Amazoníasuroriental colombiana: Medio río Caquetá (sector de Araracuara), río Igaraparaná (en la cuenca del río Putumayo) y Alto Amazonas (en el sur del Trapecio amazónico colombiano). Por otra parte, se recopiló información climatológica disponible de 8 estaciones meteorológicas en las tres cuencas, y esta información se tabuló y procesó para tener un registro de por lo menos tres décadas de los principales factores del clima: niveles del río, precipitación y temperatura.

La encuesta semi-estructurada aborda tres temas principales: percepción de cambios en el clima, adaptaciones a estos cambios y marco institucional local y nacional para enfrentar sus efectos. En este artículo me circunscribo a los resultados del primer grupo de preguntas, que tienen que ver con la percepción local del clima y una aproximación a los efectos directos de ciertos cambios climáticos sobre el modo de vida local. Las preguntas que orientaron esta parte de la entrevista fueron: ¿Cómo debería ser el clima y las épocas, según su conocimiento $\mathrm{y}$ experiencia? ¿Cómo ve el comportamiento del clima en estos últimos años? ¿Los niveles del río se han comportado normalmente o se han presentado alteraciones? ¿Con respecto a la lluvia, ha habido verano suficiente, cómo ha sido la época de lluvias? ¿Qué puede decir sobre el friaje, cómo ha sido en los últimos años? ¿Qué puede decir de los trabajos de chacra (tumba, quema, siembra), de pesca, de fructificación de frutos silvestres? ¿Cómo ha estado la salud suya, de su familia y su gente; cómo ha sido el comportamiento de las enfermedades? Hay que anotar que no todos los puntos fueron tocados con igual profundidad por todos los entrevistados, y algunos se excedieron, aportando detallados recuentos, por ejemplo de aspectos mitológicos y rituales, o se extendieron comentando sobre la situación actual de la gente.

Las 18 personas entrevistadas pertenecen a las siguientes comunidades: en el río Amazonas: Leticia, Puerto Nariño, El Castañal, San Sebastián, Kilómetro 11 y Ronda; en el río Caquetá: Araracuara y Peña Roja; y en el río Putumayo y afluentes: La Chorrera y Cordillera. Estas informaciones fueron tabuladas y organizadas alrededor de tres temas principales: percepciones de cambios en temperatura, estacionalidad y precipitación; efectos directos de estos cambios en la vida silvestre y humana; y efectos sobre la salud humana.

Esta información cualitativa fue comparada con los 
datos obtenidos de 8 estaciones meteorológicas a lo largo de las tres cuencas. Estas estaciones son administradas por el Instituto de Hidrología, Meteorología y Estudios Ambientales (Colombia, IDEAM, 2008). Se obtuvieron los registros mensuales de niveles medios del río, precipitación media mensual y temperaturas medias y máximas mensuales, para todos los períodos disponibles, de las siguientes estaciones: En el río Amazonas: Aeropuerto Vásquez Cobo, Leticia $\left(4^{\circ} 12^{\prime} \mathrm{S}, 6^{\circ} 57^{\prime} \mathrm{W}\right.$ - 1970-2007), Bocatoma $\left(04^{\circ} 11^{\prime} \mathrm{S}, 69^{\circ} 57^{\prime} \mathrm{W}\right.$ - 1990-2001) y Nazaret (0407' S 7003' W - 2000-2006); en el río Caquetá: Araracuara $\left(0^{\circ} 37^{\prime} \mathrm{S}, 7^{\circ} 24^{\prime} \mathrm{W}\right.$ - 1979-2007), Las Mercedes $\left(0^{\circ} 32^{\prime} \mathrm{S}, 72^{\circ} 10^{\prime} \mathrm{W}\right.$ - 1987-2005) y La Pedrera (01 ${ }^{\circ} 18^{\prime} \mathrm{S}, 6^{\circ} 37^{\prime} \mathrm{W}$ - 1984-2007); y en el río Putumayo: Puerto Leguízamo $\left(00^{\circ} 19^{\prime} \mathrm{S}, 7^{\circ} 46^{\prime} \mathrm{W}\right.$ $1976-2007)$ y Puerto Arica $\left(02^{\circ} 08^{\prime} \mathrm{S}, 71^{\circ} 44^{\prime} \mathrm{W}-1986-\right.$ 2007).

Estos dos conjuntos de información fueron analizados y cotejados con información secundaria y con el conocimiento previo de las tres regiones, y se presentan a partir de ahí los siguientes resultados preliminares sobre efectos y percepciones del cambio climático en esta parte de la Amazonía.

\section{RESULTADOS}

Hay que subrayar que en Amazonía se presentan dos regímenes estacionales definidos por el Ecuador climático (paralelo $2^{\circ} \mathrm{S}$ ): régimen norte y régimen sur. $\mathrm{El}$ régimen norte es común para la mayor parte de la Amazonía colombiana y regiones vecinas del Amazonas venezolano y del norte del Brasil; el régimen sur abarca el Trapecio amazónico colombiano, y es compartido por la mayor parte de la Amazonía peruana y brasileña. Estos regímenes estacionales diferenciados hacen que las alteraciones climáticas no sean uniformes para todas las áreas, como lo precisaremos abajo.

\section{INCREM ENTO DE TEM PERATURA}

Varios entrevistados de las tres cuencas están de acuerdo en que desde el año 2000 se ha sentido un incremento notorio de la temperatura. El incremento de temperatura lo sienten particularmente las mujeres que tienen que trabajar en las chacras, campos abiertos que reciben plenamente la irradiación solar. Muchas mujeres y hombres nos cuentan que deben regresar de sus chacras bien antes del mediodía, debido al fuerte calor que se siente.

Las temperaturas en Amazonía tienen un ciclo anual de variación, con temperaturas más bajas hacia la mitad del año, que corresponde al invierno del hemisferio austral. Estas percepciones son parcialmente corroboradas por los datos climatológicos locales(ver Figura 1). En las tres cuencas, el comportamiento de las temperaturas medias mensuales para el período 2000-2007, en comparación con los promedios históricos se encuentra por encima de los promedios históricos, pero dentro de un rango de la medida de desviación estándar para una muestra. Esto quiere decir que esa elevación de temperatura no sería estadísticamente significativa. Para Puerto Leguízamo (río Putumayo), los datos de temperatura del período 2000-2007 muestran un incremento promedio de $0,5^{\circ} \mathrm{C}$ en todos los meses con respecto a los promedios históricos (1976-2007). Para Leticia (río Amazonas) y Araracuara (río Caquetá), el promedio del período 2000-2007 no muestra incrementos significativos con respecto al promedio histórico (1970-2007), a excepción de la estación seca: octubrenoviembre(Leticia) y enero-febrero (Araracuara). Sin embargo, si miramos los registros de temperaturas medias mensuales para el año 2005, vemos en los tres casos, eventos ubicados por encima del primer rango de desviación. En Puerto Leguízamo observamos elevaciones significativas ese año en los meses de enero, mayo-agosto, noviembre y diciembre; en Leticia los datos de temperatura para el año 2005 registran un incremento de casi $1^{\circ} \mathrm{C}$ con respecto a los promedios históricos, en todos los meses del año, pero sólo son significativamente altos los registros de los meses de enero, mayo, junio, septiembre y noviembre; en Araracuara, los registros del 2005 sólo existen para los meses de junio a diciembre, con un comportamiento bastante anómalo: en junio la temperatura media supera en dos rangos de desviación el promedio histórico, mientras que en los meses de septiembre a diciembre son significativamente más bajos que los promedios disponibles (1980-2007).

\section{CAMBIOS EN LA ESTACIONALIDAD}

La sucesión anual de estaciones es de la mayor importancia para la gente indígena. Este ritmo ordena los tiempos del ciclo hortícola y de las prácticas rituales que ayudan a prevenir enfermedades y a promover bienestar humano, y es crucial para la reproducción de la vida silvestre. Los indígenas conciben el ciclo anual como establecido por el "Padre Creador" desde el principio de los tiempos. Los indígenas conocen bien los fundamentos ecológicos y éticos de este ciclo y de los efectos adversos de su alteración.

Este ciclo anual ideal es afectado por variaciones interanuales recurrentes. Cada número de años ocurren inundaciones excepcionalmente altas (llamadas "conejeras"), y es normal que las estaciones se adelanten o se atrasen a los tiempos previstos. Los 
indígenas están acostumbrados así a cambios en los ritmos estacionales y observan la aparición de marcadores ecológicos y fenológicos que anuncian la sucesión estacional. Lo que es crucial para la vitalidad del sistema, según los indígenas, es la presencia de las dos estaciones de viento durante el friaje y durante el verano grande, y la inundación y descenso oportunos de los ríos, acoplado con la sucesión de estaciones lluviosas y secas. Todo esto garantiza la reproducción exitosa de los peces y de la fauna terrestre, la maduración de las frutas, y el ciclo ordenado de actividades hortícolas y rituales. En los últimos años, sin embargo, los marcadores ecológicos han venido ocurriendo de forma muy anticipada o retrasada, o des-sincronizados con la estación que marcaban. Enseguida comento los principales cambios observados.

\section{ESTACIONES SECAS Y LLUVIOSAS}

Un comentario generalizado es que la precipitación y las estaciones lluviosas están desorganizadas; no hay estaciones secas o de lluvias claramente demarcadas: "Llueve cuando no debe llover, hace calor cuando no debe hacer calor", afirma un hombre indígena de Araracuara. Ya no hay más una estación seca claramente marcada con vientos fuertes; hay calor pero no acoplado con los vientos. Hay sucesión de días secos, pero tiene lugar fuera de la época prevista del año: julio-agosto en el régimen sur, enero-febrero en el régimen norte.

La Figura 2 nos ilustra estas percepciones para algunos años anómalos en las tres cuencas, es decir aquellos donde los registros de precipitación están por fuera de un rango de desviación estándar. Los datos de precipitación en Araracuara para el año 2007 nos muestran que la precipitación fue significativamente baja durante los meses de abril y octubre, significativamente alta para el mes de junio, y significativamente muy alta para el mes de julio. Los datos de La Pedrera son significativamente bajos para mayo y significativamente altos para noviembre de 2005. Y en Leticia los datos del 2005 son significativamente bajos para febrero, junio, julio y septiembre, y significativamente muy altos para octubre.

\section{FLUCTUACIONES ESTACIONALES DE LOS NIVELES DE LAS AGUAS}

Los niveles del río, que son el marcador estacional más prominente, han mostrado toda suerte de alteraciones. Un caso notable que nos muestra una clara correlación entre las percepciones de los indígenas y los datos meteorológicos lo registramos en el río Caquetá. Los indígenas de la región de Araracuara me narraron que en el 2005 el río inundó los rebalses en el mes de mayo y los peces pusieron sus huevos; al mes siguiente, sin embargo, el río descendió abruptamente antes de que los peces estuvieran suficientemente maduros, causando su muerte. Ese mismo año, el comportamiento anómalo de los niveles del río afectó la reproducción de las tortugas, particularmente de la tortuga gigante Podocnemisexpansa (charapa), una especie amenazada, que requieren la aparición oportuna de las playas para colocar sus huevos. Cuando el río desciende y aparecen las playas, en el mes de octubre (régimen norte), las tortugas hacen una primera postura en las playas. Normalmente, esta primera postura es arrastrada por un ascenso final de las aguas (el "lavaplayas"); entonces, las tortugas hacen su postura definitiva a medida que el río sigue descendiendo y entra la estación seca. En 2005, en el río Caquetá, según narraron los indígenas, la segunda postura de las tortugas también fue arrastrada por un ascenso anormal del río.

Estas dos alteraciones de los niveles del río que afectaron la reproducción de la fauna acuática son claramente corroboradas por los datos climáticos de ese año (véase Figura 3). Los datos de la estación Araracuara para ese año nos muestran que el río Caquetá subió de nivel de marzo a mayo por encima de los promedios históricos, pero dentro de un rango de desviación estándar; en junio inició un descenso anticipado, y en julio alcanzó un nivel significativamente bajo (más de dos rangos de desviación estándar). Estos datos se correlación perfectamente con la observación de los indígenas en cuanto a la muerte de los peces. En agosto el río volvió a ascender y mantuvo niveles normales entre agosto y octubre, pero en noviembre, cuando hacen su postura las charapas en el régimen norte, tuvo un ascenso significativamente alto. Los datos de La Pedrera (400 $\mathrm{km}$ aguas abajo sobre el mismo río Caquetá) nos muestran un comportamiento similar de los niveles, con excepción del descenso abrupto en el mes de julio. Entre estas dos localidades, el río Caquetá recibe las aguas de por lo menos dos grandes afluentes de origen amazónico: los ríos Yarí y Mirití; esto nos indicaría que el descenso del río sentido en Araracuara fue debido a un evento de disminución de caudales de los afluentes de origen andino.

\section{SEQUÍA DEL 2005}

El año 2005 fue un año excepcionalmente seco en la Amazonía meridional y occidental. Fuertes emisiones de humo proveniente de extensos incendios que ocurrieron tan lejos como el occidente de Brasil y el sur de Bolivia, alcanzaron a llegar hasta el río Amazonas (Marengo et al., 2008 muestran que la sequía del 2005 no estuvo ligada a un evento de El 
Niño, sino a temperaturas superficiales más altas en el Océano Atlántico tropical). En las regiones bajo el régimen norte, esta sequía no se sintió, sino que causó alteraciones en los niveles del río, como se notó arriba.

La Figura 4 muestra cómo la precipitación en los regímenes norte y sur estuvo exactamente invertida en el 2005. Los datos de precipitación de Leticia ese año registran precipitaciones medias significativamente bajas en los meses de junio a septiembre, y significativamente muy altas en el mes de octubre. En cambio, en Puerto Leguízamo, sobe el río Putumayo y afectado por el régimen climático norte, la precipitación fue normal durante todo el año, con niveles de precipitación altos de marzo a julio.

\section{OTRAS PERCEPCIONES}

Dos cosechas prominentes, que marcan el pico de la estación seca (Bactrisgasipaes, la palma de pijuayo o chontaduro)y de la estación fría (Mauritia flexuosa, la palma de aguaje o canangucho) han mostrado un comportamiento anormal en la mayoría de los años recientes, según reportan los indígenas.

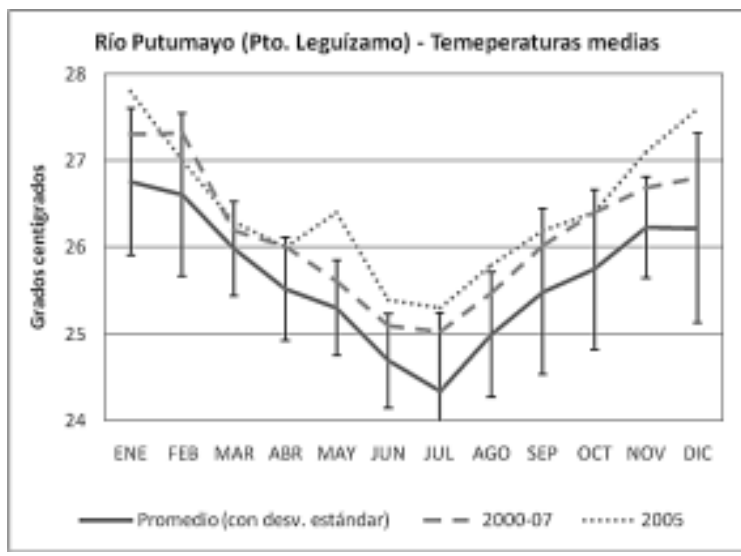

El friaje o estación fría está teniendo lugar por adelantado, es menos fuerte o es muy corto. Los vientos del friaje no alcanzan a purificar los árboles, que no llegan a madurar sus frutos. Estos vientos se conciben como colectores de las enfermedades del monte; si no llegan con suficiente fuerza, la contaminación se acumula y afecta la salud natural y humana. Un hombre ticuna de Río Amazonas lo expresó de manera sucinta: "Los ancianos dicen que al Padre del friaje lo han matado."

Otro efecto sentido de los cambios climáticos recientes ha sido sobre la salud. La mayoría de las personas entrevistadas ha subrayado la ocurrencia creciente de enfermedades respiratorias e intestinales, no sólo más fuertes, sino también de nuevos tipos no conocidos antes. Las enfermedades transmitidas por vectores, como malaria y dengue, al parecer no han tenido incrementos, pero el carácter cambiante de la fluctuación de los ríos pueden crear condiciones favorables para la reproducción de vectores de maneras aún imprevisibles.

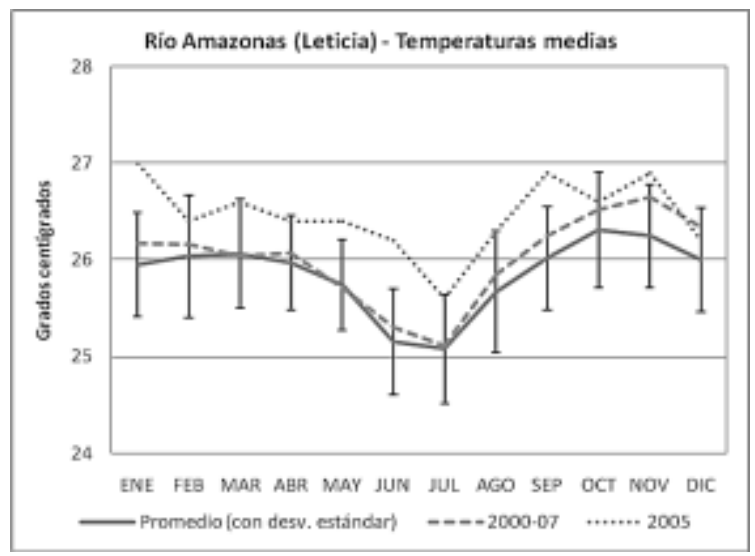

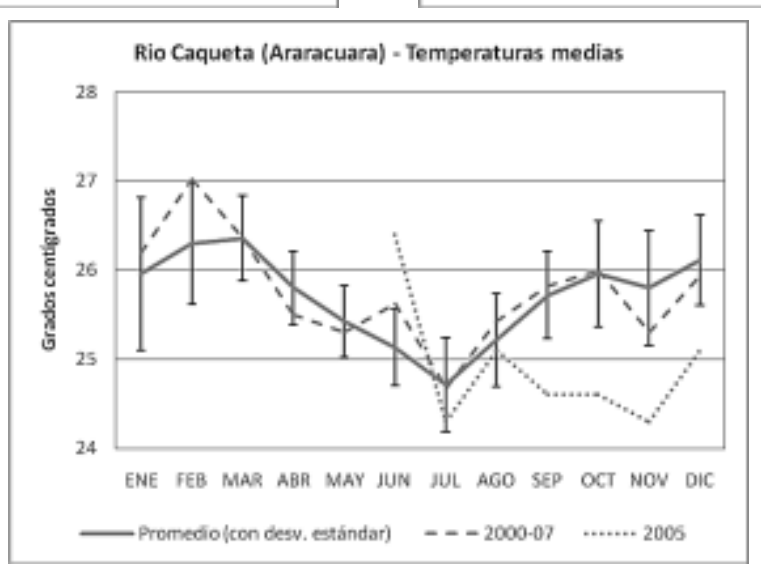

Figura 1. Temperaturas medias mensuales. Elaborada a partir de datos de la Estación Puerto Leguízamo $\left(00^{\circ} 19^{\prime} S\right.$, 74²46'W), 1976-2007; Estación Aeropuerto Vásquez Cobo (Leticia) (04¹2' S, 6957'W), 1970-2007; y Estación Araracuara $\left(0^{\circ} 37^{\prime} \mathrm{S} 72^{\circ} 24^{\prime} \mathrm{W}\right)$, temperaturas medias mensuales para los años 1980-2007. (Colombia, IDEAM , 2008). 

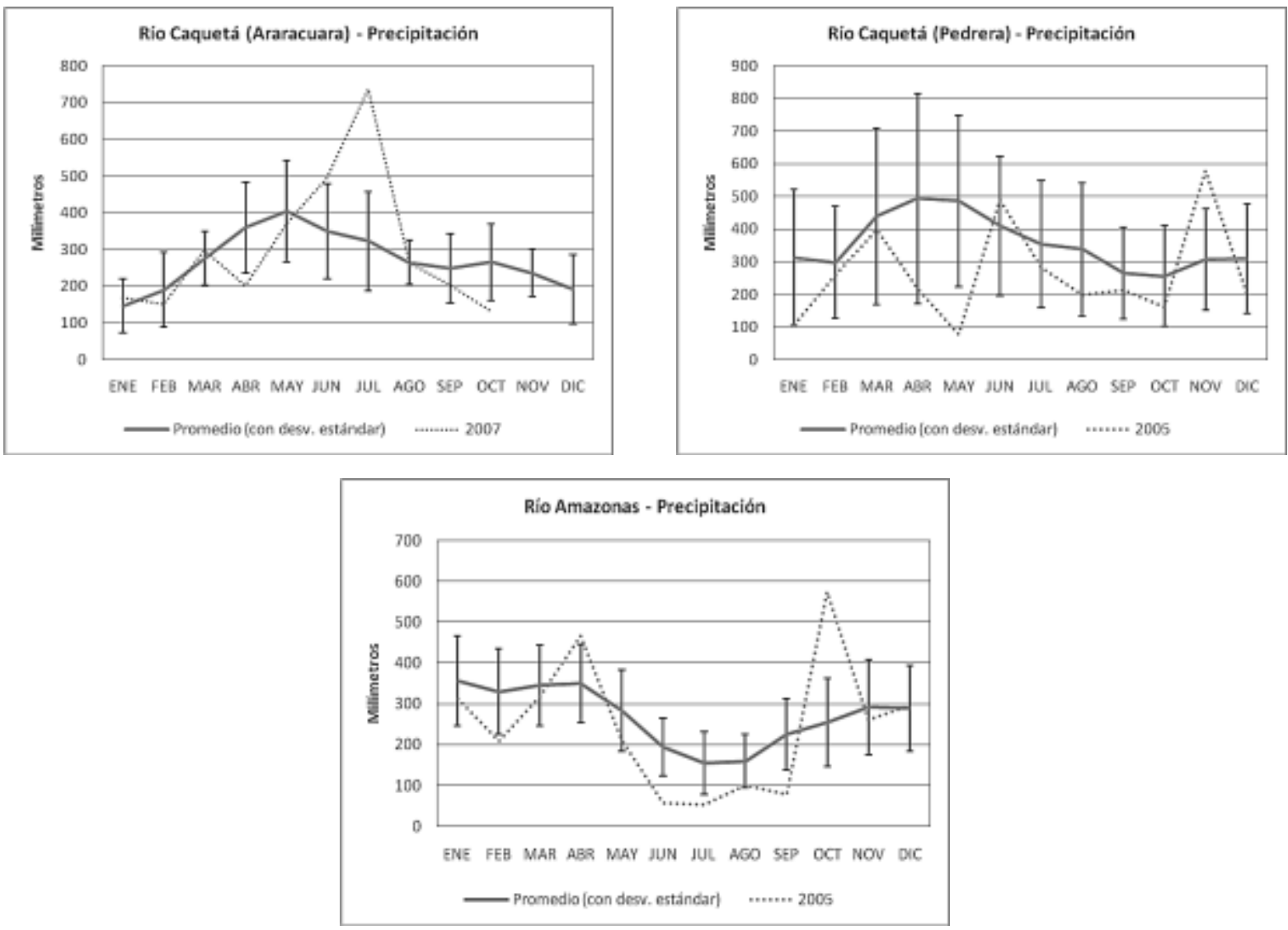

Figura 2. Precipitación media mensual. Elaborada a partir de datos meteorológicos de la Estación Araracuara $\left(0^{\circ} 37\right.$ 'S $72^{\circ} 24^{\prime} W$ ), 1979-2007; Estación La Pedrera (01ํ18' S, 69³7' W), 1984-2007; y Estación Aeropuerto Vásquez Cobo (Leticia) (4¹2'S, 6957'W), 1970-2007. (Colombia, IDEAM , 2008).
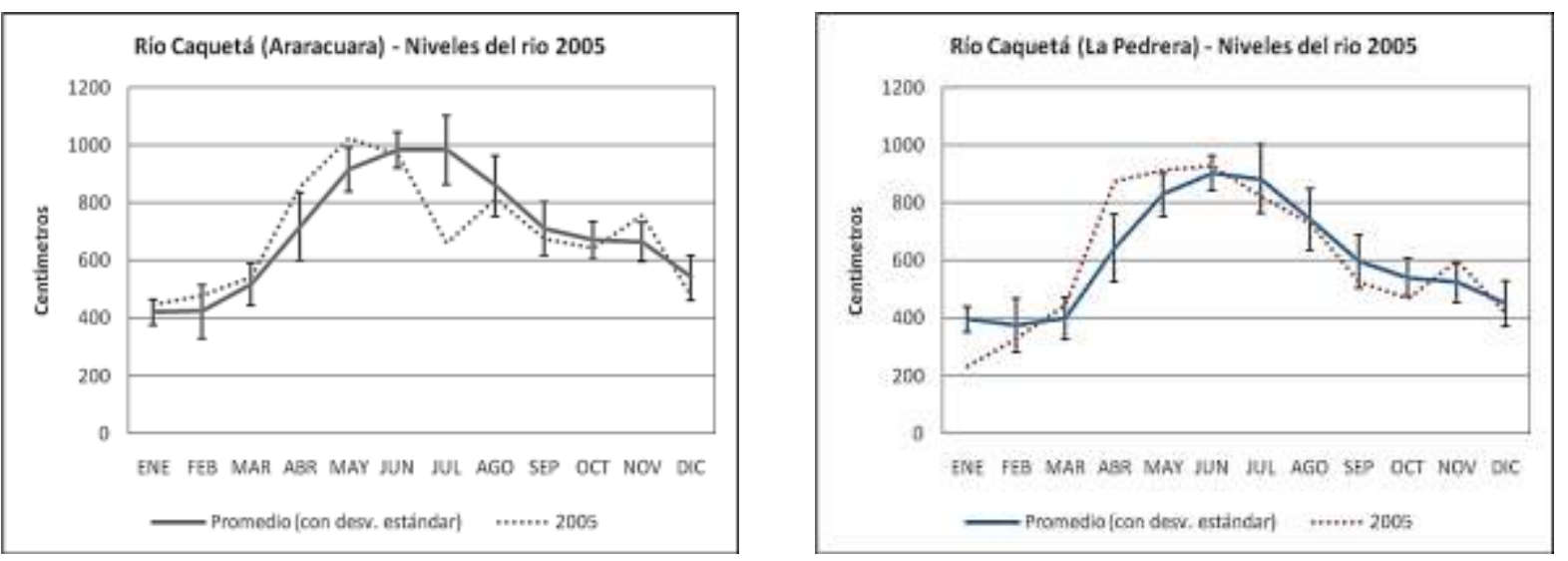

Figura 3. Niveles del río Caquetá 2005. Elaborada a partir de datos de la Estación Las M ercedes (0³2'S 72¹0'W ), 1987 2005; y Estación La Pedrera (01ํ1' S, 69³7' W ), 1984-2007. (Colombia, IDEAM , 2008). 

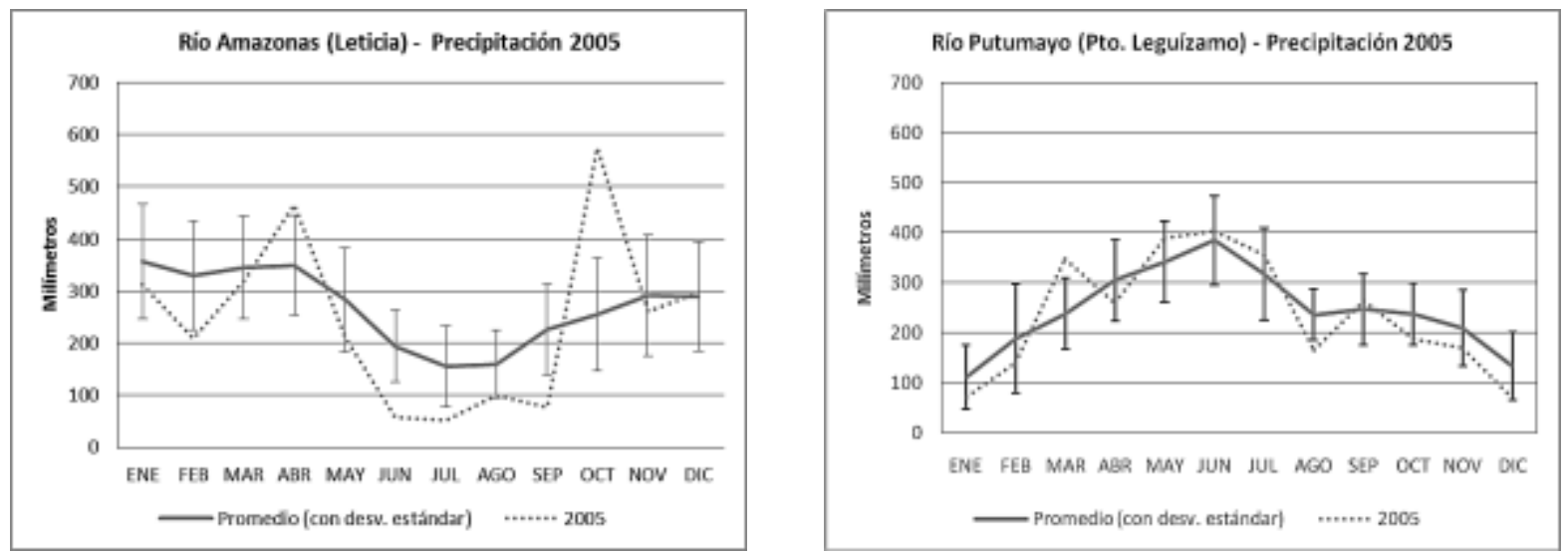

Figura 4. Precipitación 2005. Elaborada a partir de datos meteorológicos de la Estación Aeropuerto Vásquez Cobo

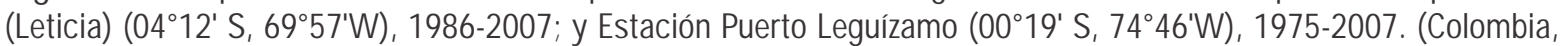
IDEAM , 2008).

\section{DISCUSIÓN Y CONCLUSIONES}

La primera conclusión que obtenemos de estas observaciones y datos es que, más que la elevación de la temperatura de la atmósfera, son los efectos sobre el régimen estacional amazónico y sobre la vida silvestre y humana los que causan los cambios más impredecibles y de mayor impacto. De hecho, los datos de temperatura no nos revelan eventos significativos, a excepción de algunos años excepcionalmente secos, como el 2005, y esto sólo para el régimen sur.

\section{IM PACTOS SOBRE LA VIDA SILVESTRE}

Un primer impacto de la alteración de la estacionalidad es sobre la fauna y la flora. Como presentamos en los resultados, pulsos de inundación del río que no alcanzan a inundar las varzeas o igapós, que tiene descensos y ascensos abruptos, afectan directamente la reproducción de la fauna acuática, particularmente de los peces, que constituyen la fuente de proteína más importante para la gente amazónica, o de especies amenazadas como las tortugas del género Podocnemis. Friajes cortos, débiles o fuera de tiempo, junto con la sucesión errática de estaciones secas y lluviosas hacen que la floración y maduración de los frutales silvestres ocurra fuera de tiempo o fracasen por completo, como fue observado por los indígenas. La alteración de los ciclos fenológicos de los frutales tiene un impacto sobre la reproducción de los peces y sobre la fauna terrestre, que se alimenta de esas frutas, y sobre los humanos, que se alimentan de ambos.

\section{IM PACTOS SOBRE EL SISTEM A DE HORTICULTURA}

Los cambios en los regímenes de precipitación y estacionalidad impactan directamente el crecimiento temprano y el éxito de los cultivos. Algunas cosechas necesitan ser replantadas dos o tres veces. La yuca, la cosecha más importante en las chacras indígenas, es más resistente al calor y a la sequía y crece bien en suelos pobres. Esto garantiza una fuente de carbohidratos, pero amenaza la diversidad de cosechas en las huertas.

Los indígenas de esta parte de la Amazonía trabajan por lo menos tres tipos de chacras: (1) chacras tumbadas en bosque maduro sobre suelos de terra firme (suelos ácidos y arcillosos, pobres en nutrientes), que requieren un período de sequía más largo y necesitan ser quemados para mejorar el $\mathrm{pH}$ y enriquecer el suelo; éstas son los tipos de chacras con mayor diversidad cultivada; (2) chacras tumbadas en bosque secundario, que requieren un período más corto de sequía, pero son menos productivas y más propensas a la invasión de malezas; éstas son chacras de menor duración y menos diversas; y (3) chacras en llanuras aluviales sobre suelos cuaternarios, que se enriquecen con las inundaciones periódicas; éstas no requieren quema, pero sirven solamente para cultivar especies que pueden ser cosechadas antes de que la llanura aluvial sea nuevamente inundada; éstas son las chacras más productivas pero las menos diversas. Los tres sistemas de cultivo han sido afectados directamente por los cambios recientes en los regímenes de estacionalidad, aunque de diferente manera. 
Las chacras tumbadas en bosque maduro dependen de la ocurrencia regular de una estación de días secos, calientes y con vientos constantes, que garanticen una quema completa de la parcela. La no ocurrencia de una estación de verano seco en los últimos años ha ocasionado que las parcelas tumbadas se quemen de forma incompleta o no puedan ser quemadas. En el primer caso (quema incompleta), los indígenas tienen que picar y amontonar la madera que no se quemó para hacer hogueras y terminar el proceso de quema, intensificando de esta manera el tiempo de trabajo invertido en la chacra. Este tipo de chacras en bosque maduro es trabajado principalmente por los grupos indígenas de la Amazonía nororiental colombiana, que tienen abundante acceso a bosque maduro; la gente indígena más cercana a áreas urbanas o de la colonización ya no hace este tipo de chacras, pues su acceso al bosque maduro está seriamente restringido. Estas chacras de alta diversidad sobre monte firme son importantes no sólo como fuente del alimento, sino también para la vida ritual y ceremonial. Así, tanto la diversidad cultivada como la vitalidad del ceremonial en las áreas más tradicionales se ven directamente afectadas por el cambio climático.

Por otra parte, chacras más productivas pero menos diversas sobre suelos aluviales son amenazadas por cambios imprevisibles del nivel del río. Inundaciones súbitas o tempranas pueden destruir cosechas enteras. Este tipo de chacras son hechas sobre todo por gente ribereña, quien vive a lo largo de los ríos principales de aguas blancas (Amazonas, Putumayo) y también como alternativa para los grupos tradicionales del río de Caquetá. Se utilizan para sembrar cultivos para la venta y para la subsistencia. La inestabilidad de esta forma de cultivo, debido a patrones cambiantes e impredecibles de los niveles del río, a largo plazo, afecta la economía de subsistencia y de mercado de grupos menos tradicionales, y también formas alternativas de horticultura de grupos tradicionales.

Las chacras sobre bosque secundario - menos diversas que aquellas en bosque maduro y menos productivas que aquellas sobre suelos aluviales - aparecen entonces como la alternativa más confiable. Este tipo de chacras ha llegado a ser habitual para aquellos grupos con territorios restringidos, y es una alternativa tanto para la gente ribereña como para los grupos tradicionales de tierra firme. Estas chacras demandan menos trabajo y no requieren de una estación seca prolongada para quemarse con éxito. La tumba repetida del bosque secundario, sin embargo, conduce a la degradación de los suelos y la creciente invasión de malezas.

\section{IM PACTOS SOBRE LA SALUD}

Los indígenas reconocen la recurrencia estacional de enfermedades virales, y recurren a prácticas preventivas de naturaleza tanto material como espiritual. La aparición y desaparición de enfermedades recurrentes (gripes, diarreas) está muy relacionada con los pulsos de inundación, los regímenes de precipitación y los cambios de temperatura. La enfermedad, en la visión de los grupos del Caquetá-Putumayo, está en los árboles y en el agua; la inundación y los vientos del friaje sirven para colectar toda esa enfermedad del mundo y drenarla hacia el mar cuando los ríos descienden. Cuando la inundación no es suficientemente alta o el friaje no es fuerte, la enfermedad se acumula en la atmósfera y en las aguas, haciendo que los trastornos respiratorios e intestinales se prolonguen y aparezcan otros nuevos. Especialistas rituales de la región del medio Caquetá (régimen norte) expresan claramente que las prácticas y prevenciones rituales que han servido para prevenir y controlar los efectos adversos de cada estación, cada vez está teniendo menos efecto, debido al desorden de las estaciones.

Otros efectos sobre la salud humana - no evidentes aún, pero predecibles - tienen que ver con la potencial disminución de las fuentes de alimento. Los peces constituyen la principal fuente de proteína para las poblaciones humanas, y son uno de los recursos más directamente afectados por la alteración en el régimen de estacionalidad. Los impactos sobre el sistema de chacras afecta tanto la disponibilidad de carbohidratos (pequeñas chacras en bosque secundario no producen la cantidad de yuca para una familia) y la diversidad de otros tubérculos y frutos cultivados (las chacras son menos diversas), que enriquecen y complementan la dieta. Fuentes alternativas de proteína (fauna terrestre, avifauna, insectos) y de vitaminas y minerales (frutas silvestres) son también directamente afectadas.

\section{GÉNERO, EDADYSITUACIÓN SOCIAL}

Aunque la mayor parte de estos cambios tiene efectos para todas las categorías de género y edad, algunos grupos son más vulnerables a impactos actuales y potenciales. Además, dependiendo de las condiciones ecológicas (régimen norte, régimen sur; piedemonte, selva oriental) y socio-cultural (acceso a territorio, recursividad cultural, contacto) se configuran escenarios de impacto diferenciales.

Los infantes y niños son el grupo más vulnerable a enfermedades virales. También, la disminución en la disponibilidad de proteína y las fallas en las cosechas afectan directamente el crecimiento y desarrollo sano de los segmentos más jóvenes de la sociedad.

Los especialistas rituales, quienes dependen en gran 
medida de producción de comida cultivada abundante y diversa y de amplio acceso a recursos de cacería, son impactados por la dificultad creciente de establecer chacras sobre monte firme que son requeridas para llevar a cabo las actividades rituales. También, enfermedades nuevas y más fuertes menoscaban sus capacidades de curación y prevención.

Las mujeres en edad adulta reciben la mayor carga de impacto con estos cambios, al ser las mujeres quienes tradicionalmente están a cargo del trabajo hortícola. El trabajo en parcelas abiertas bajo temperaturas crecientes, la pérdida de cosechas, la necesidad de replantar y los rendimientos menores de las cosechas afectan su salud y autoestima. Las chacras en bosque secundario o chacras en bosque maduro que no queman suficientemente incrementan el tiempo requerido para erradicar malezas o para completar manualmente el proceso de quemado. Además, la mala salud y la desnutrición de los niños afectan directamente el trabajo y las responsabilidades de las mujeres.

Hombres jóvenes, que son responsables por la adquisición de proteína y la apertura de nuevas chacras, son afectados por disminución en la disponibilidad de los recursos de pesca. El esfuerzo para conseguir cacería terrestre y pescado aumenta para ellos, y los criterios para decidir los tiempos correctos para tumbar y quemar chacras se vuelven más inciertos.

Los grupos indígenas con mayor autonomía territorial, que derivan su sustento sobre todo de recursos del bosque y del agua, y mantienen una vida ritual activa, son los más fuertemente afectados en su subsistencia. Estos grupos valoran grandemente las chacras en bosque maduro plantadas con gran variedad de especies, dependen fuertemente de la pesca y la cacería como fuente de proteína, y cuidan su salud en buena medida con sus propios medios y conocimientos. Su subsistencia se basa en su capacidad de interpretar los ciclos naturales regulares y de actuar en consecuencia. Ciertamente, estos grupos tienen contacto con la sociedad nacional, están incorporados en alguna medida a la economía de mercado y tienen acceso a los servicios de salud y educación públicas, pero un porcentaje grande de su subsistencia depende de su conocimiento, uso y manejo de los recursos del bosque y del agua. Estos son los grupos más conscientes de estos cambios y también los más vulnerables a sus efectos. En esta categoría están los grupos indígenas del área de Vaupés (familias lingüísticas Tukano oriental, MakuPuinave y Arawak), de la región de CaquetáPutumayo (familias lingüísticas Witoto, Bora-Miraña y Andoke), de la región de Guainia (familias lingüístsicasMaku-Puinave y Arawak) y de algunos grupos del Trapecio amazónico (familias lingüísticas Tikuna, Pebas-Yagua y Tupi).

Un escenario distinto ocurre con los grupos indígenas con autonomía territorial restringida y poco o ningún acceso al bosque maduro, que dependen de la horticultura en bosque secundario, cosechas de pan coger sobre suelos aluviales, pesca comercial, trabajo asalariado, turismo y venta de las artesanías para su subsistencia. Ellos se ven afectados por los cambios mencionados arriba, en la medida que utilizan los recursos del río y del bosque, y son igualmente afectados por las enfermedades y los incrementos de temperatura. Estos grupos indígenas están conscientes del calendario estacional (o restringido a esos aspectos que afectan directamente sus actividades), su conocimiento tradicional es más limitado y los especialistas rituales por lo general tienen un papel menos central en la sociedad. Su mayor acceso al mercado y a los servicios de salud y educación públicas les ofrece recursos para amortiguar los impactos sobre su subsistencia. Son menos conscientes y menos vulnerables que los grupos más tradicionales, pero el efecto combinado del cambio climático tiende a acelerar la incorporación al trabajo asalariado, la integración al mercado, la migración hacia áreas urbanas y la pauperización. En esta categoría están los grupos indígenas en las zonas de colonización del piedemonte andino y a lo largo del río de Guaviare, y los grupos indígenas de las otras áreas que viven en la vecindad de áreas urbanas.

\section{BIBLIOGRAFÍA CITADA}

Bunyard, P. 2008. Why Climate is Dependent on Biodiversity.In: Zarate, C.; Ahumada, C. (Eds.).Fronteras en la globalización: Localidad, biodiversidad y comercio en la Amazonía. Bogotá: Fundación Konrad Adenauer, Pontificia Universidad Javeriana, pp. 21-42

Colombia, IDEAM (Instituto de Hidrología, Meteorología y Estudios Ambientales). 2008. Sistema de información ambiental. (http://institucional.ideam.gov.co/)

Marengo, J. A.; Nobre, C. A.; Tomasella, J.; Cardoso, M. F.;Oyama, M. D. 2008. Hydro-climatic and ecological behaviour of the drought of Amazonía in 2005.Philosophical Transactions of the Royal Soc i e ty B, 363: $1773-1778$. (http://journals.royalsociety.org/content/238x8181 0815588k/fulltext.pdf)

Salick, J.; Byg, A. 2007.Indigenous Peoples and Climate Change.Tyndall Center for Climate $\mathrm{Ch}$ ange Re search, Oxford. (http://www.tyndall.ac.uk/publications/Indigenou speoples.pdf). 